\title{
THE ENVIRONMENT OF $z>1$ 3CR RADIO GALAXIES AND QSOS: FROM PROTO-CLUSTERS TO CLUSTERS OF GALAXIES?
}

\author{
J. P. Kotyla ${ }^{1}$, M. Chiaberge ${ }^{1,2}$, S. Baum ${ }^{3}$, A. Capetti $^{4}$, B. Hilbert ${ }^{1}$, F. D. Macchetto ${ }^{1}$, G. K. Miley ${ }^{5}$, C. P. O’Dea ${ }^{3}$, \\ E. S. Perlman ${ }^{6}$, W. B. Sparks ${ }^{1}$, and G. R. Tremblay ${ }^{7}$ \\ ${ }^{1}$ Space Telescope Science Institute, 3700 San Martin Drive, Baltimore, MD 21218, USA; marcoc@stsci.edu \\ ${ }^{2}$ Center for Astrophysical Sciences, Johns Hopkins University, 3400N. Charles Street, Baltimore, MD 21218, USA \\ ${ }^{3}$ University of Manitoba, Deptb. of Physics and Astronomy, 66 Chancellors Cir., Winnipeg, MB R3T 2N2, Canada \\ ${ }^{4}$ INAF-Osservatorio Astronomico di Torino, Via Osservatorio 20, I-10025 Pino Torinese (TO), Italy \\ ${ }^{5}$ Universiteit Leiden, Rapenburg 70, 2311 EZ Leiden, The Netherlands \\ ${ }^{6}$ Florida Institute of Technology, 150 W University Blvd, Melbourne, FL 32901, USA \\ ${ }^{7}$ Yale University, Department of Astronomy, 260 Whitney Ave, New Haven, CT 06511, USA \\ Received 2016 January 8; revised 2016 April 15; accepted 2016 May 9; published 2016 July 19
}

\begin{abstract}
We study the cluster environment for a sample of 21 radio loud active galactic nuclei from the $3 \mathrm{CR}$ catalog at $z>1,12$ radio galaxies (RGs) and nine quasars, with Hubble Space Telescope (HST) images in the optical and IR. We use two different approaches to determine cluster candidates. We identify the early-type galaxies (ETGs) in every field by modeling each of the sources within a $40^{\prime \prime}$ radius of the targets with a Sèrsic profile. Using a simple passive evolution model, we derive the expected location of the ETGs on the red sequence (RS) in the colormagnitude diagram for each of the fields of our sources. For seven targets, the model coincides with the position of the ETGs. A second approach involves a search for over densities. We compare the object densities of the sample as a whole and individually against control fields taken from the GOODS-S region of 3D-HST survey. With this method we determine the fields of ten targets to be cluster candidates. Four cluster candidates are found by both methods. The two methods disagree in some cases, depending on the specific properties of each field. For the most distant RG in the 3CR catalog (3C 257 at $z=2.47$ ), we identify a population of bluer ETGs that lie on the expected location of the RS model for that redshift. This appears to be the general behavior of ETGs in our fields and it is possibly a signature of the evolution of such galaxies. Our results are consistent with half of the $z>1$ RGs being located in dense, rapidly evolving environments.
\end{abstract}

Key words: galaxies: active - galaxies: clusters: general - galaxies: elliptical and lenticular, $\mathrm{cD}$ - galaxies: jets quasars: general

\section{INTRODUCTION}

Radio galaxies (RGs) and radio loud quasars (QSOs) are among the most energetic phenomena in the universe. The hosts of these objects at low redshifts $(z<0.3)$ are massive $\left(M \sim 10^{11} M_{\odot}\right)$ giant elliptical galaxies (Zirbel 1996; Donzelli et al. 2007). Their powerful jets are believed to be produced by rapidly spinning supermassive black holes (Blandford \& Znajek 1977; Wilson \& Colbert 1995; Ghisellini et al. 2014). There is also growing evidence that their black hole masses are above $\sim 10^{8} M_{\odot}$ (Laor 2000; Dunlop et al. 2003; Best et al. 2005; Chiaberge \& Marconi 2011; Calderone et al. 2013; Castignani et al. 2013; Mao et al. 2015). Radio loud (RL) active galactic nuclei (AGNs) are typically found in rich Mpc-scale environments. At low redshifts $z<0.3$ the fraction of RGs that reside in clusters is as high as 70\% (Zirbel 1997) and a large fraction of them are located in the cluster $\mathrm{cD}$ galaxy. At higher redshifts $(z \sim 0.5$ and above) the fraction of RL AGNs in clusters is $\sim 50 \%$ (Prestage \& Peacock 1988; Hill \& Lilly 1991; Best 2000; Galametz et al. 2012; Wylezalek et al. 2013). However, due to the lack of study of statistically meaningful samples for $z>1$, the exact fraction of RGs residing in clusters at these redshifts is still not firmly known. This is in fact a central question not only for our understanding of the physics of the RL AGN phenomenon, but also for high- $z$ cluster searches. In fact, high- $z$ RGs are often used as beacons for clusters or proto-clusters at $z>2$ (Miley \& De Breuck 2008, for a review).
A Hubble Space Telescope (HST) Cycle 20 snapshot program (GO13023, P.I. Chiaberge, M., Hilbert et al. 2016) was designed to study the environment of a sample of 3CR (Spinrad et al. 1985) RGs and QSOs at $z>1$ in much greater detail. In particular, one of the central goals of the project was to determine the fraction of clusters in the high- $z$ 3CR sample. Some of the most commonly used methods for determining the presence of clusters are based on the X-ray emission from the intracluster medium (Rosati et al. 2002), the SunyaevZel'dovich effect (Sunyaev \& Zeldovich 1972), the red sequence (RS) method (Gladders \& Yee 2000), as well as the search for over densities of galaxies through a number of statistical tools (Castignani et al. 2014, and references therein). The RS technique is known to identify clusters out to a redshift of $z \sim 2$. Such a method is based on a pattern found in colormagnitude diagrams (CMDs) due to the passive evolution of early-type galaxies (ETGs).

In this paper we use two different approaches to study the Mpc-scale environment of 3CR sources and determine their possible association with clusters or groups. First, we focus on investigating the presence of an RS in the field of each target. Second, we compare the density of objects in each field against the average density of a control sample. The plan of the paper is as follows: in Section 2 we describe the sample and the HST observations; in Section 3 we discuss our method to detect objects and perform photometry; Section 4 focuses on detailing the methods we use to assess the presence of a cluster and 
Table 1

The Observed Sample

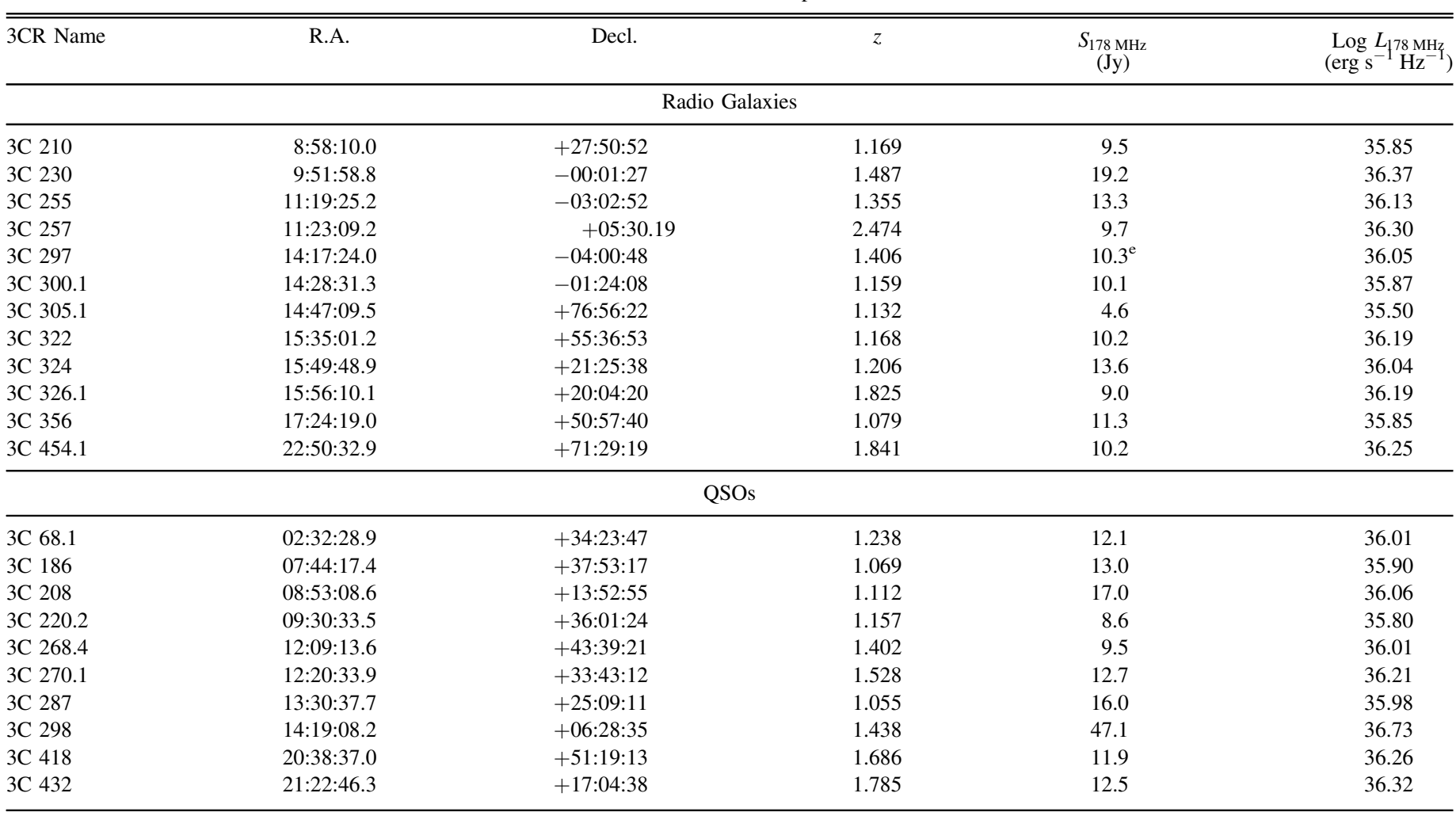

Note. In Column 1 of the table we give the names of the objects. In Columns 2 and 3 we display the coordinates. In Column 4 we show redshifts. In Column 5 we show the flux at $178 \mathrm{MHz}$, and in Column 6 we show the logarithm of the luminosity.

describes the results; and in Section 5 we discuss our findings. Lastly, in Section 6 we draw conclusions.

Throughout the paper we use the AB magnitude system and a $\Lambda$ CDM cosmological model with the following parameters: $H_{0}=70 \mathrm{~km} \mathrm{~s}^{-1} \mathrm{Mpc}^{-1} ; \Omega_{m}=0.27 ; \Omega_{\Lambda}=0.73$.

\section{OBSERVATIONS AND DATA REDUCTION}

Our targets were observed in optical and near-IR with HST's Wide Field Camera 3 (WFC3) between 2012 December and 2013 May as part of snapshot program GO13023. HST snapshot surveys of complete samples are well suited to statistical studies, since the observations are scheduled by randomly picking objects from the original target list to fill gaps in the HST schedule. The proposal originally planned for the complete sample of $583 \mathrm{CR} z>1$ targets and throughout Cycle 20 we obtained data for 22 of these 58 . The observed sample represents $38 \%$ of our proposed sample. Of the 22 observed targets, 12 objects are RGs and 10 are QSOs. The observed sample spans a redshift range of $1.05<z<2.47$.

The names and properties of all the observed targets are listed in Table 1.

In the case of $3 \mathrm{C} 418$, the source is at low galactic latitude and thus the field is contaminated by a large number of stars. The field is also heavily reddened $\left(\mathrm{A}_{V}=2.9\right.$ Hilbert et al. 2016) making the analysis of the environment of this high- $z$ object impossible. For this reason, we choose to exclude this target from the discussion of this paper.

Both the UVIS $(F 606 W)$ and IR $(F 140 W)$ channels of WFC3 were used to image each of our targets. The UVIS observations have a field of view of $162^{\prime \prime} \times 162^{\prime \prime}$ with a pixel scale of $0 . " 04$. The IR observations cover a field of view of $123^{\prime \prime} \times 136^{\prime \prime}$, corresponding to a projected distance of $1.0 \mathrm{Mpc}$ $\times 1.2 \mathrm{Mpc}$ at $z=1.5$. The pixel scale for WFC3 IR is 0 !" 13 .

We first download the data from the Mikulski Archive for Space Telescopes. We customize the data reduction for our data set using two different reductions for the UVIS and IR images. In the custom reduction for the UVIS data, we first correct for charge transfer efficiency losses using the algorithm derived by Anderson \& Bedin (2010), which produces the "FLC" (flat fielded and CTE corrected) calibrated files. The second part of the custom reduction focuses on the cosmic ray removal through a multi-step process. To this aim, we use the Python version of L. A. Cosmic (van Dokkum 2001) twice on each image. The first run is made with conservative parameters, in order to make sure that only the obvious and brighter cosmic rays are removed, and no real objects are affected. A second L. A. Cosmic run is then performed with more stringent parameters to remove the cosmic rays in the region around the chip gap only. This is needed since we only have two dither points, and therefore while the chip gap is fully covered, there is a region of the gap that is only imaged once. At least two images are needed for the Astrodrizzle cosmic ray removal task to work effectively. Astrodrizzle is then used to combine the images, and remove residual cosmic rays. However, with only two dither points, we noticed that not all events are completely removed. In particular, pixels that are impacted by cosmic rays in both images cannot be corrected. In order to remove these residual cosmic rays we first make a mask that includes pixels showing significant flux excess compared to the surrounding pixels. These are identified by a simple algorithm that 
compares each drizzled image with both the difference and the ratio of the original two images. The marked pixels in the mask are then grown using a Gaussian kernel of appropriate FWHM (generally $\sim 1$ pixel), in order to fix a slightly larger area. Pixels in that area are then replaced by linear interpolation of the surrounding pixels using the IRAF task fixpix.

The reduction for the IR data uses the standard HST pipeline followed by a persistence correction (Long et al. 2011). For more details regarding the steps of the custom reduction, see Hilbert et al. (2016).

\section{PHOTOMETRY}

We first identify sources on the IR images and then we perform photometry on both IR and UVIS images based on the object catalog derived from the IR. The emission of each galaxy in the UVIS images is dominated by a younger stellar population since the rest-frame wavelength of the UVIS pass band $(F 606 W)$ resides in the UV at $z>1$. These young stellar components usually appear as "blobby" structures (i.e., regions of star formation), and do not allow a straightforward identification of the object as a whole. At $1<z<2.5$ the IR pass band $(F 140 W)$ is rest-frame optical and thus samples older stellar populations, resulting in a smooth regular shape. For this reason, we use the IR images to identify sources.

The procedure is as follows. We use Source Extractor (SExtractor) (Bertin \& Arnouts 1996) in the MAG BEST mode for the IR images. Such a mode allows for measurements of the flux for sources with different morphologies. When set in this mode, SExtractor uses a flexible elliptical aperture around every detected object and measures all of the flux inside that, provided that the aperture is larger enough. SExtractor constructs the specific elliptical apertures using the Kron radius, described in Kron (1980). However, if the elliptical aperture is smaller than 3 pixels in radius, SExtractor defaults to a circular aperture of 3 pixels. Also, if the contribution form other sources is determined to exceed $10 \%$, an isophote corrected flux/magnitude is used. This method retrieves the fraction of flux in the wings of an object that would be missed in the isophotal magnitudes by assuming a Gaussian profile. For full technical details see Bertin \& Arnouts (1996) Section 7.4.2.

In order to select the optimal parameters we simulate a 512 $\times 512$ pixel image convolved with a Tinytim (Krist et al. 2011) model point-spread function consisting of 78 galaxies representative of the galaxies within our targets' fields. We run SExtractor on this image with varying parameters (Kron factor, and minimum radius) until we minimized the difference between the known, simulated magnitudes and those given as output by SExtractor. After establishing the ideal parameters, Kron factor of 2.5 pixels and minimum radius of 3.5 pixels, we run SExtractor on the IR images to identify sources.

We use the SExtractor catalog produced from the IR data to select the regions on which we perform aperture photometry on the UVIS images. As noted above, since the galaxies in the UVIS images are more likely to be irregularly shaped, we use aperture photometry to measure the flux of all the components located within the region covered by the galaxy in the IR image. The aperture size is adopted from the output of the SExtractor fit for the IR sources. The specific radius used for each source is $r=\alpha \times R_{.9}$ where $R_{.9}$ is the $90 \%$ effective light radius of the corresponding IR source. After testing a range of values on simulated galaxies of different morphologies, we find that $\alpha=1.2$ is optimal to accurately measure the magnitude of the objects in the IR band. This value is also appropriate to encompass all of the flux of the individual components seen in the UVIS image that are co-spatial to a single source in the IR data. After we complete the photometry we correct for galactic absorption and work with the AB magnitudes ( $F 606 W$ zero point 26.0691, F140W zero point 26.4524).

The final step in producing the source photometric catalog for each field is to remove objects identified as stars so that the photometric data for each field contains only galaxies.

\section{METHODS FOR FINDING CLUSTERS AND GROUPS}

We use two complementary methods to establish which of our fields may contain a cluster or group. Each approach has limitations and so in order to find the best candidates, we compare the results from both methods.

First we look for the presence of an RS in CMDs in analogy with the so-called RS technique (Gladders \& Yee 2000). This method is sensitive to massive clusters where the RS is more clearly defined. Second we identify over densities of objects in the field of our targets when compared with the object densities from randomly selected fields. By comparing the results from multiple methods, we can better identify which fields are the best cluster candidates. Below we describe the two different approaches.

\subsection{Red Sequences}

The existence of an RS in a CMD is a known indicator of clustering. A cluster's RS is identified as a linear relationship in CMD where bright (early-type) galaxies, dominated by an old stellar population, are located. The position (color and slope) of the RS is determined by the evolution of the cluster ETGs and thus depends on redshift. Therefore, to determine the presence of an RS, we first identify the early-type objects and determine whether their positions on the CMD coincide with the expected position of the RS at the redshift of the radio source in that field.

\subsubsection{Morphological Classification}

The first step in determining whether the CMD exhibits a cluster RS is to determine which objects in the field are ETGs. We morphologically classify all galaxies inside a 40" (346 kpc at $z=1.5$ ) radius encircled upon the target.

To classify the galaxy morphologies, we fit the IR 2D surface brightness profiles of all galaxies in each field using a Sèrsic law in Galfit (Peng et al. 2010). The Sèrsic law is given as

$$
\Sigma(r)=\Sigma_{e}\left[-\kappa\left(\left(\frac{r}{r_{e}}\right)^{1 / n}-1\right)\right]
$$

where $\Sigma_{e}$ is the surface brightness at the effective radius $r_{e}$ (half of the total flux is within $r_{e}$ ) and the Sèrsic index is $n$. We classify a galaxy as early-type if the best fit results in a Sèrsic index, $n$, of $2<n<8$. For each ETG we visually inspect the image as well as the residuals in order to determine if the fit was appropriate. In a small number of cases $(<2 \%)$ Galfit misclassifies an object due to low signal-to-noise, or contamination from nearby objects. Figure 1 shows an example of an 


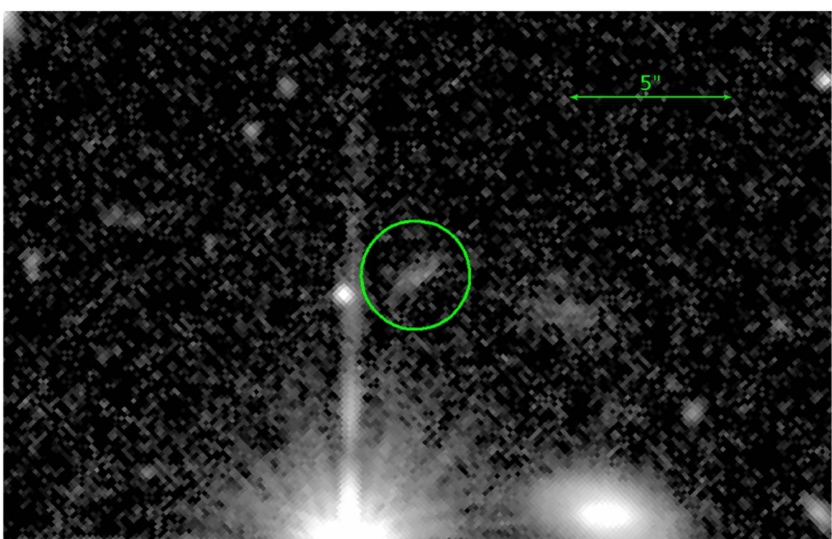

Figure 1. WFC3 IR $F 140 \mathrm{~W}$ image of an object in the field of $3 \mathrm{C} 68.1$. This object was originally fit and estimated to have a Sèrsic index within our earlytype range. After visual inspection, it is clear that this object is in fact not an ETG and should not be marked as so in our CMD.

object classified as early-type that we reject possibly due to low signal-to-noise or contamination.

\subsubsection{RS Modeling}

In order to produce a model RS estimate, we use GalEv (Kotulla et al. 2009), the evolutionary synthesis modeling program. The GalEv input parameters include mass, metallicity, and redshift of formation. For our models, we assume a single burst of star formation followed by passive evolution. Our goal is not to provide a detailed model of the evolution of single galaxies, but rather a comparison of different evolutionary states at the redshifts of our targets. Therefore a simplified framework with a single star burst and passive evolution is sufficient. Furthermore, we tested that more complicated evolutionary models with non-instantaneous or multiple star-forming events would not provide significant changes for the location of the predicted RSs unless the starforming events are very recent. GalEv outputs the modeled magnitudes starting from the redshift of formation.

As a reference we use the observed RS in the well-studied X-ray selected cluster RDCS 1252.9-2927 at $z=1.24$ (Blakeslee et al. 2003). The cluster was observed with HST/ ACS using the filters $F 775 \mathrm{~W}$ and $F 850 L P$ as part of program GTO/ACS 9290 and is known to have a well-defined RS even at such a high redshift. We derive the parameters that produce the best representation of the observed RS for two different redshifts of formation $\left(z_{1}=6.5, z_{2}=20\right)$. We model the evolutions of galaxies with two different masses (i.e., corresponding to different magnitudes), which allow us to identify the slope in the CMD. The choice of parameters (mass and metallicity), are consistent with mass-metallicity relations described in Lee et al. (2008). The derived set of parameters is then used to obtain the model magnitudes at each redshift for the two filters used in our WFC3 observations. The parameters used for the model are listed in Table 2.

In Figures 2, 3 we show the resulting color ( $F 606 W-$ $F 140 \mathrm{~W}$ ) plotted against the $F 140 \mathrm{~W}$ magnitude for each of the objects with $m_{F 140 W}<27$ within a $40^{\prime \prime}$ radius surrounding the targets. Objects marked with green circles indicate ETGs. Blue circles indicate that we originally classify the object as earlytype but establish that the object was misclassified after visual inspection (as described in Section 4.1.1). In addition, we
Table 2

GalEv Parameters

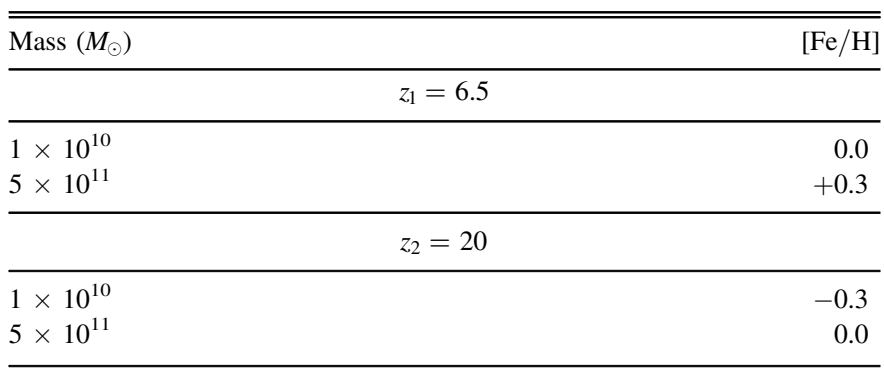

Note. For the redshifts of formation $(6.5,20)$, each row shows the GalEv parameters used to generate a galaxy used in our fit. Other parameters that remain consistent across all GalEv models are IMF: Salpeter IMF (.1-100 $\left.M_{\odot}\right)$; burst: no burst; type: E (elliptical); and extinction law: none.

display the two model RSs corresponding to the two redshifts of formation ( $z_{1}$ in black, $z_{2}$ in red). Given the uncertainties of the models and considering the range of redshift spanned by our sources, we highlight an area corresponding to a spread of \pm 0.3 mag where we qualitatively expect to observe an RS.

Using the CMD, we define a cluster candidate as any field in which we observe at least half of the galaxies classified as early-type lying within the area spanned by the models. We count all objects whose $1 \sigma$ error bar falls within the \pm 0.3 mag band around either one of our two RS models.

One of our targets (namely 3C 210) is known to reside in a well-studied high-redshift cluster characterized by the presence of an RS (Stanford et al. 2002). 3C 186 is also known to reside in a cluster, but in that case the cluster was confirmed by the clear detection of X-ray emission from the intracluster medium (Siemiginowska et al. 2010). We use these clusters to test the reliability of our RS method. Reassuringly, this method correctly identifies both fields as cluster candidates because of the presence of a substantial number of ETGs within the region of the CMD where the corresponding model RSs lie.

\subsubsection{RS Results}

Using this classification scheme we find seven cluster candidates associated with three QSOs and four RGs. The results of this analysis are summarized in Table 3. For each field, we report the number of objects falling within the $40^{\prime \prime}$ radius around the target $\left.\left(n_{40}\right)\right)$, the number of objects within the region that are classified as early-type $\left(n_{\mathrm{ETG}}\right)$, and the number of ETGs whose $1 \sigma$ error bar falls within the \pm 0.3 color band around each of the RS models with redshifts of formation 6.5 and $20\left(\mathrm{ETG}_{1}\right.$ and $\mathrm{ETG}_{2}$ respectively). The objects classified as cluster candidates based on the RS method described above are marked in Column 6 with "RS."

In addition to the seven candidates identified with the RS method, we also point out that two additional fields (3C 220.2 and 3C 356, see Figures 2 and 3, respectively) show a significant number of ETGs lying within the area spanned by the models. Our method does not identify these objects as cluster candidates because of the presence in the same field of a large number of bluer ETGs that do not fall close to the RS models. In 3C 356 such ETGs are relatively bright, thus it is possible that at least some of them are foreground objects. Only spectroscopy of these objects can address this issue.

Another object that shows an interesting population of blue ETGs is 3C 257 (see Figure 3), our highest redshift target 

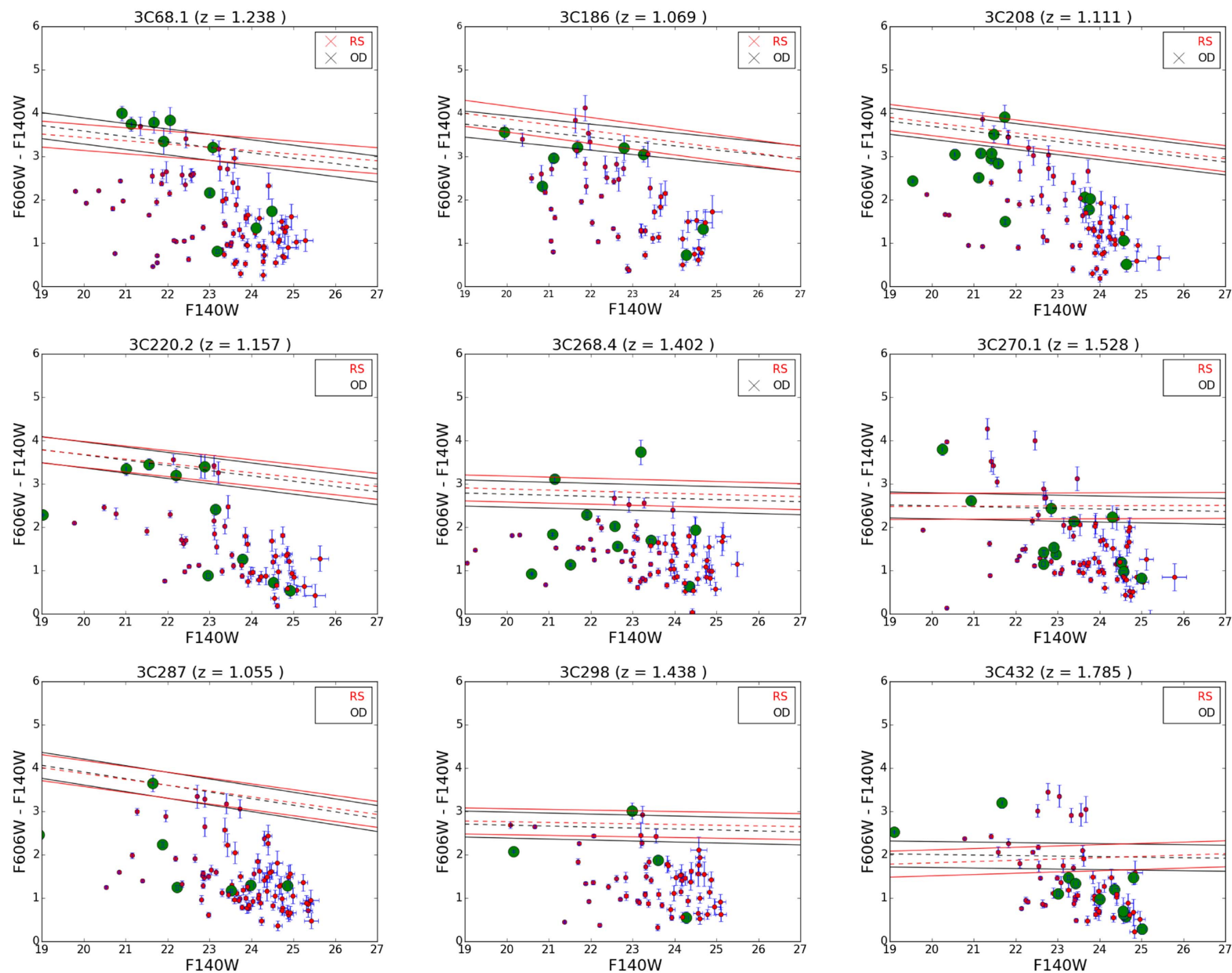

Figure 2. CMD of the nine QSOs in our sample. The plots contain all objects with magnitude less than 27 contained within a $40^{\prime \prime}$ radius of the target. The green circles indicate that we classify the object as early-type, while blue circles represent objects that we originally classify as early-type but later reject due to contamination or another anomaly. The red and black lines represent our model RSs using GalEv parameters with redshifts of formation of 20 and 6.5, respectively. The dashed lines surrounding the models visualize a spread of $\pm 0.3 \mathrm{mag}$. The target QSOs are not displayed in the figures.

$(z=2.474)$. Interestingly, these blue ETGs lie exactly on top of the RS models. This will be further discussed in Section 5.1.

\subsection{Over densities}

\subsubsection{Method}

In addition to the method using RSs described above, we investigate the existence of an over density of galaxies in the regions surrounding the targets. The presence of over densities could be an indication of clustering. Our method to search for significant over densities compares the object counts in a region within $40^{\prime \prime}$ of each target against the average density of objects in control fields. In the range of redshift between 1 and 2.5, the projected size corresponding to the radius we adopt changes by about $6 \%$ (the smallest and largest size being approximately $347 \mathrm{kpc}$ at $z=1.6$ versus $326 \mathrm{kpc}$ at $z=1$, for the adopted cosmology). However, the main concern is that the cluster core size might undergo a significant evolution between $z=1$ and $z$ $=2.5$. Because of the poorly understood relationship between cluster size and redshift, we prefer to keep the radius fixed, for the sake of simplicity.

The control fields are derived from a sample of 36 nonoverlapping regions covered by the 3D-HST Survey data in the GOODS-S area (Brammer et al. 2012). Such a region was imaged using WFC3 IR and the $F 140 \mathrm{~W}$ filter, i.e., the same configuration used in our 3CR observations. The selected regions in the $3 \mathrm{D}-H S T$ data are chosen to avoid gaps present in the mosaic image. We create a catalog of objects in such regions using SExtractor. We manually remove any objects detected by SExtractor that are the result of artifacts.

In order to ensure the completeness of the two samples, we select objects with $m_{F 140 W}<24.5$ mag in both our $3 \mathrm{CR}$ fields and the 3D-HST images. The upper bound of $24.5 \mathrm{mag}$ is derived from the $\log N(<m)$ versus $m_{F 140 W}$ plot for our sample as well as the comparison fields in the 3D-HST image. Figure 4 is a modified version of the well known $\log N-\log S$ diagnostic, which allows us to determine at which flux (or magnitude) a survey becomes incomplete. In Figure 4 the red dots represent 

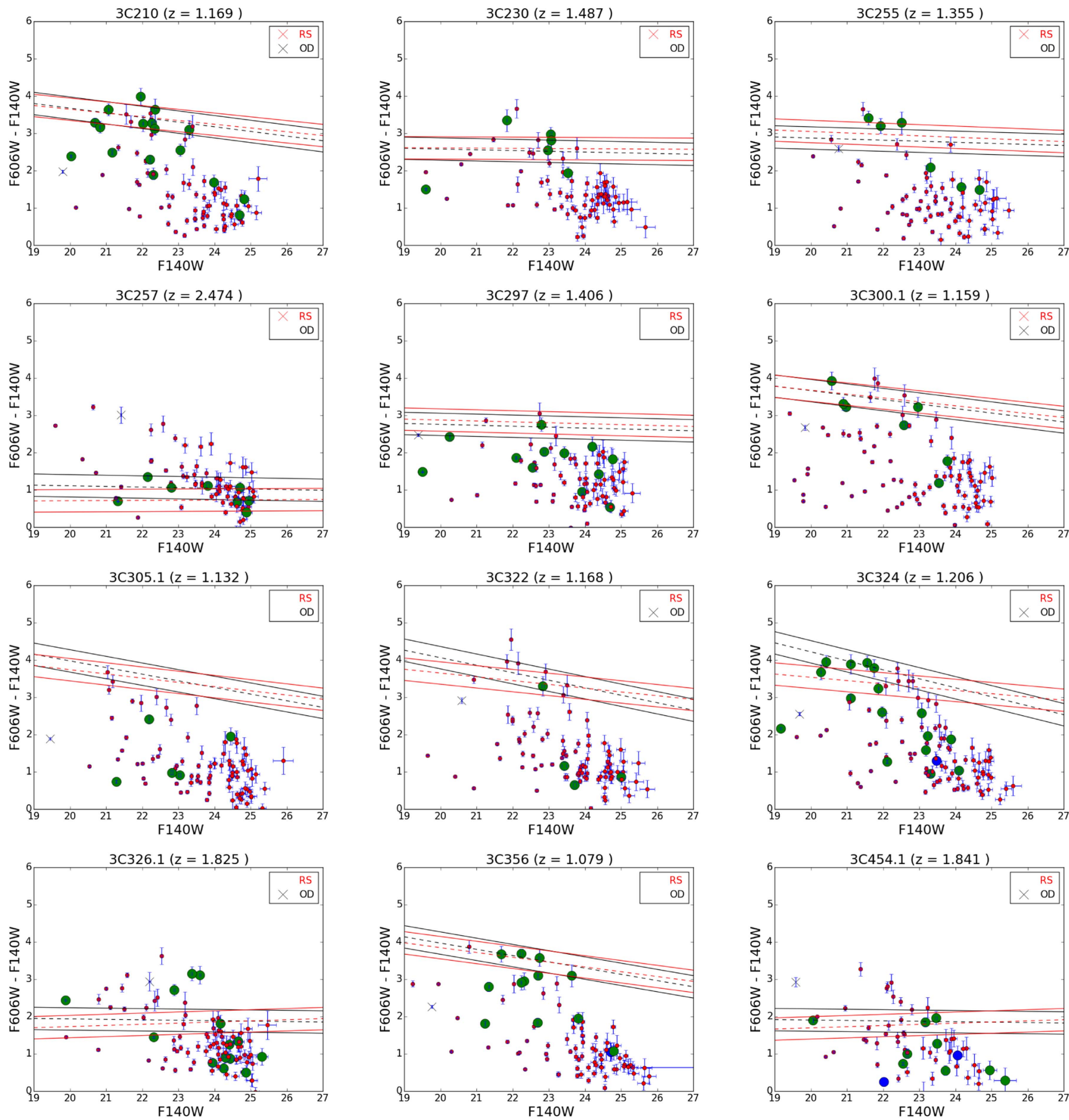

Figure 3. CMD of the 12 RGs in our sample. The plots contain all objects with magnitude less than 27 contained within a $40^{\prime \prime}$ radius of the target. The green circles indicate that we classify the object as early-type, while blue circles represent objects that were originally misclassified as early-type but later rejected due to contamination or another anomaly (see Section 4.1.1). The red and black lines represent our model RSs using GalEv parameters with redshifts of formation of 20 and 6.5 , respectively. The dashed lines surrounding the models visualize a spread of \pm 0.3 magnitudes. A blue " $\mathrm{X}$ " represents the $3 \mathrm{CR}$ target.

the cumulative source distribution of the entire GOODS-S field covered by 3D-HST, while the blue dots are the data from our sample. In the figure we shift the 3CR data downward by 2 on the $\log N(<m)$ axis ( $y$-axis) in order to better display both sets of data, which otherwise overlap.

We see that significant deviations from the fitted lines in both cases occur for magnitudes fainter than 24.5. This is expected, since the exposure time of the 3D-HST pointings is only slightly longer than that of the $3 \mathrm{C}$ SNAPSHOT data.

\subsubsection{Over densities: Results}

First we test whether the RL AGNs in our sample lie in overdense regions on average. Figure 5 presents the histograms for 
Table 3

Summary of Classification Methods

\begin{tabular}{|c|c|c|c|c|c|}
\hline 3CR Name & $n_{40^{\prime \prime}}$ & $\begin{array}{l}n_{\mathrm{ETG}} \\
\end{array}$ & $\mathrm{ETG}_{1}$ & $\overline{\text { ETG }_{2}}$ & Cluster Candidate \\
\hline \multicolumn{6}{|c|}{ QSOs } \\
\hline 3C 68.1 & 90 & 9 & 5 & 5 & RS, OD \\
\hline 3C 186 & 78 & 8 & 4 & 4 & RS, OD \\
\hline $3 \mathrm{C} 208$ & 89 & 15 & 3 & 2 & OD \\
\hline 3C 220.2 & 50 & 10 & 3 & 4 & No \\
\hline $3 C 268.4$ & 79 & 11 & 1 & 1 & OD \\
\hline $3 \mathrm{C} 270.1$ & 75 & 12 & 4 & 4 & No \\
\hline $3 \mathrm{C} 287$ & 70 & 7 & 1 & 1 & No \\
\hline 3C 298 & 52 & 4 & 1 & 1 & No \\
\hline $3 \mathrm{C} 432$ & 65 & 12 & 0 & 0 & No \\
\hline \multicolumn{6}{|c|}{ RGs } \\
\hline $3 \mathrm{C} 210$ & 90 & 17 & 9 & 9 & RS, OD \\
\hline $3 \mathrm{C} 230$ & 74 & 6 & 3 & 3 & $\mathrm{RS}$ \\
\hline $3 \mathrm{C} 255$ & 77 & 6 & 2 & 3 & $\mathrm{RS}$ \\
\hline $3 \mathrm{C} 257$ & 50 & 8 & 7 & 7 & $\mathrm{RS}$ \\
\hline 3C 297 & 64 & 12 & 2 & 1 & No \\
\hline 3 C 300.1 & 99 & 7 & 4 & 4 & RS, OD \\
\hline $3 C 305.1$ & 70 & 5 & 0 & 0 & No \\
\hline $3 \mathrm{C} 322$ & 81 & 4 & 1 & 1 & OD \\
\hline 3 C 324 & 81 & 15 & 5 & 5 & OD \\
\hline $3 C 326.1$ & 79 & 14 & 1 & 1 & OD \\
\hline $3 \mathrm{C} 356$ & 73 & 12 & 5 & 5 & No \\
\hline 3C 454.1 & 84 & 9 & 3 & 4 & OD \\
\hline
\end{tabular}

Note. The first column shows the name of the $3 \mathrm{CR}$ target for a particular field In Column 2 we report the number of objects falling within the $40^{\prime \prime}$ radius around the target $\left(n_{40^{\prime \prime}}\right)$. In Column 3 we show the number of objects within the region that are classified as early-type $\left(n_{\mathrm{ETG}}\right)$. Columns 4 and 5 refer to the number of ETG whose $1 \sigma$ error bar falls within the $\pm 0.3 \mathrm{mag}$ band around each of the RS models with redshift of formation 6.5 and $20\left(\mathrm{ETG}_{1}\right.$ and $\mathrm{ETG}_{2}$ respectively). In column 6 we indicate with "RS" and/or "OD" whether the method based on the RSs or the over densities, respectively, classifies the field as a cluster candidate.



Figure 4. $\log N$-mag graph for our aggregated fields (blue dots) as well as the $3 \mathrm{D}-H S T$ control sample (red dots). The $y$-axis is the logarithm of number of objects that have magnitudes less than or equal to the corresponding value on the $x$-axis. We fit the relationships for the region $19<m_{F 140 W}<24$ with linear models and display the resulting fits (red and blue for 3D-HST and 3C samples respectively). We shift the $3 \mathrm{CR}$ data downward by 2 on the $\log N(<m)$ axis $(y-$ axis) in order to better display both sets of data, which otherwise overlap.

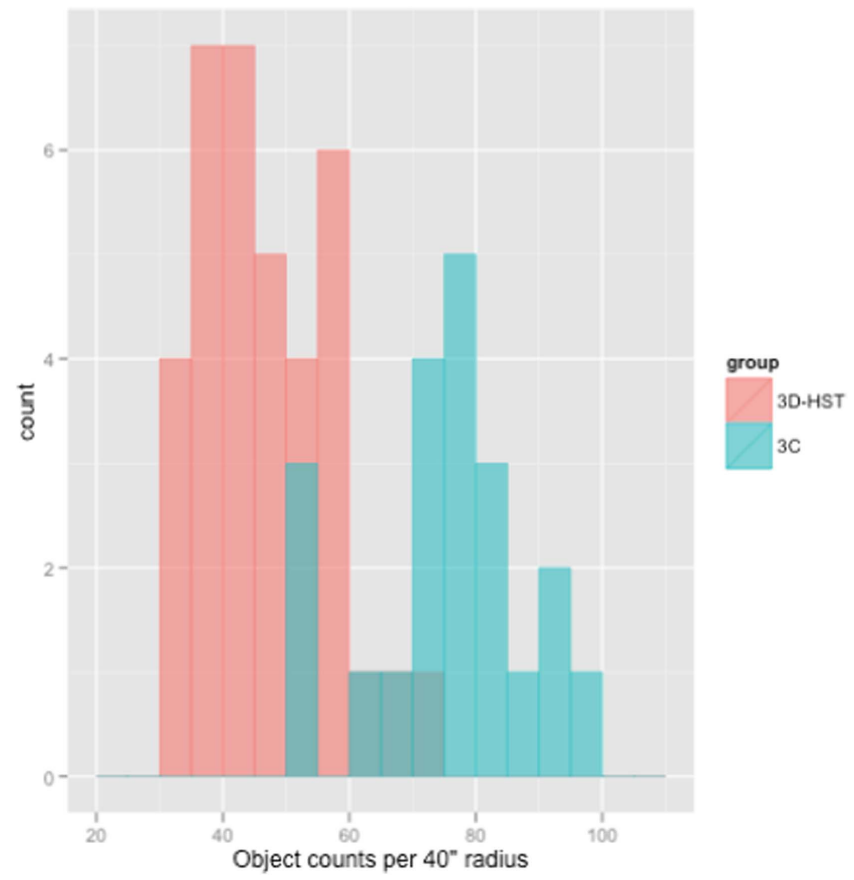

Figure 5. Distributions of the number of objects within the $40^{\prime \prime}$ radius around the radio sources (blue), and the distribution of the number of objects in the randomly selected fields within the GOODS-S region (red).

the distribution of counts among our sample (red) and the 3DHST sample regions (blue). From visual inspection it is apparent that the number density of the objects in the 3CR fields is higher than in the control sample. The mean of the object counts in the 3D-HST $40^{\prime \prime}$ radius regions is 45.9 with a standard deviation of 10.6 objects. The corresponding mean of the 3CR fields is 74.8 with a standard deviation of 13.2 objects. By comparing these two values we find that the environments of the radio sources are on average denser. This result comes from a Student's $t$-test where we are able to reject the null hypothesis, i.e., that the two mean object densities are equal. We determine that the mean of the object counts in the 3CR regions is higher than that of the control fields with very high statistical significance given by a $p$-value of $2.2 \times 10^{-10}$. The test was performed using the $\mathrm{R}$ function t.test in the stats package (R Core Team 2014).

In addition, we investigate the individual deviations of the number of objects in each of our fields from the average 3D$H S T$ object density. We find that four out of nine QSOs and six out of $12 \mathrm{RG}$ environments show an over density with $>3 \sigma$ significance, which corresponds to a $p$-value of $\sim 0.003$ for a normal distribution. In total, this amounts to $48 \% \pm 20 \%$ (the error corresponds to a 95\% Bayesian credible interval) of our sample of RL AGNs being in overdense regions. The fraction of objects that lie in over-dense regions for both the QSO and RG groups is highly uncertain due to the small sample sizes. The two fractions $\left(45 \%{ }_{-27}^{+28}\right.$ and $50 \%_{-25}^{+24}$ for the QSOs and RGs, respectively) are statistically indistinguishable.

Lastly, we perform a Bootstrap Kolmogorov-Smirnov test in order to rigorously test the difference in the distributions of the object densities in the fields of the QSOs and RGs. The result of this test is that we fail to reject the null hypothesis $(p=$ 0.904), i.e., that the distributions are different. 
The objects classified as cluster candidates based upon the presence of an over density are marked in Column 6 of Table 3 with "OD" (over density).

Note that the density of galaxies in the central region of a cluster may depend on the dynamical state of the cluster itself, and that might affect our ability to identify cluster candidates. For example, if the cluster is in a merging state, its central regions may display a less pronounced distribution of objects compared to that of a relaxed cluster. Another possible complication is that the radio source might not be exactly at the center of the cluster, in particular for structures that are not relaxed yet. However, for the specific goals of this work, we do not consider either of these scenarios in detail.

As for the RS method in Section 4.1.2, we test our overdensity method against the two confirmed clusters in our sample, which are associated with 3C 186 and 3C 210. Our second method correctly identifies both fields as cluster candidates because of the presence of a significant over density of objects around the radio source.

\section{DISCUSSION}

We used two different methods to investigate the environment of 3CR RGs and QSOs at $z>1$. The method based on the object over densities returns a larger number of cluster candidates (11) as compared to the method based on the presence of an RS (seven).

In four cases (two QSOs and two RGs, namely 3C 68.1, 3C 186, 3C 210, 3C 300.1) both methods return a positive result. As already discussed above, two of these objects are already known to reside in clusters confirmed by either the detection of X-ray emission from the intracluster medium (3C 186, Siemiginowska et al. 2010) or by the presence of a well established RS (3C 210, Stanford et al. 2002). These two clusters are identified correctly by both methods, i.e., we find both an over density of sources and a substantial number of ETGs lying on the region of the CMD where the RS is expected at the redshift of each of the two targets. The fact that those two confirmed clusters are correctly identified by both of our methods gives us confidence that our cluster candidates are robust at least for the cases in which both of our methods agree on a positive detection. However, all of our cluster candidates must be confirmed by other means, e.g., with spectroscopic information on the redshift of each cluster galaxy member.

For the remaining two objects for which both of our methods return a positive result, there is no information in the literature about any association with a cluster of galaxies, to the best of our knowledge. However, we point out that the presence of an RS is very clear from their CMDs in Figures 2 and 3. This is particularly evident in the case of 3C 68.1 in which five out of the nine ETGs lie close to the RS models.

Three RGs (3C 230, 3C 255, 3C 257) are identified as cluster candidates by the RS method but they do not show an over density of objects. Even if at least half of the ETGs fall within the area spanned by the models, an RS is not clearly identified in 3C 230, since they show no clear linear relationship. In 3C 255 only five objects appear to define an RS. 3C 257 is a peculiar case that we discuss in Section 5.1. It is possible that these three radio sources reside in small groups that include a small population of ETGs, but the evidence for the presence of a cluster is not convincing.

Six objects, two QSOs (3C 208, 3C 268.4), and four RGs (3C 322, 3C 324, 3C 326.1, 3C 454.1) show significant over densities but no evidence for an RS. In the case of 3C 322 (Figure 3) only one of the red objects that lie on the RS models is determined to be an ETG. Conversely, in the field of 3C 324 we see a relatively large number of red ETGs falling onto the models. However, this population does not represent the majority of the ETGs in the field. Thus our method does not identify this as a cluster candidate.

The remaining five QSOs and three RGs are not identified as cluster candidates according to either of our methods.

In Figure 6 we show four example fields, one for each category. In the top left panel the field of 3C 68.1 is classified as a cluster by both the RS and the over-density methods. The presence of a large number of red objects in this dense field is apparent from visual inspection. In the top right panel, the field of 3C 230 is classified as a cluster according to the RS method, but there is not a significant over density of objects. In this case, it is possible that a cluster is present, but since the object is located at $z \sim 1.5$ a significant number of the cluster galaxies are below our detection threshold. Alternatively, this object could be located in a group that includes a smaller number of galaxies with respect to rich clusters. In the lower left panel we display an example of a field (surrounding 3C 322) that shows a significant over density, but no RS. Note that despite the presence of a large number of red objects, they are not identified as ETGs, and thus the RS method fails to classify this field as a cluster. In the lower right panel we show the field of 3C 297, in which neither the RS method nor the over-density method classify this field as a cluster. The paucity of objects in this field is clear from the image.

We do not find any evidence for a statistically significant correlation between the number of galaxies detected within the $40^{\prime \prime}$ radius and redshift, or between the number of ETGs and redshift. However, there are only four sources in our observed sample at $z>1.6$. Therefore it is difficult to identify any possible trends with redshift. It is interesting to note that the most distant object 3C 257 lies at the lower end of the galaxy number count distribution, but such an object is not the one for which the $40^{\prime \prime}$ radius corresponds to the smallest projected size. It is possible, in that specific case, that the surface brightness detection limit of our observations may be playing a role. Alternatively, the smaller number count might be due to the younger age of the cluster, which may correspond to a significantly larger core radius. But a much larger number of objects should be observed in order to statistically test these possible scenarios.

As the statistical analysis shows, a fraction consistent with about one half of our targets are associated with cluster or group candidates. This is in agreement with previous results using ground-based observations. In fact, Hill \& Lilly (1991) found that about $50 \%$ of powerful 3C RGs at $z \sim 0.5$ inhabit rich clusters. Similar results were also found by Best (2000) based on ground-based near-IR observations. Two of our objects (3C 324, 3C 356) are in common with the sample presented in that work. Qualitatively, we conclude that our results are consistent with the published CMDs in that work.

\subsection{Blue ETGs in the Field of $3 C 257$}

In the case of the most distant source, 3C $257(z=2.474)$, we observe a particularly interesting feature. The derived RS models for the targets redshift predict that the ETGs should display an $F 606 W-F 140 W$ color $\sim 1$, i.e., they should fall in the "blue" region of the CMD. This is a straightforward 




Figure 6. Four fields of 3 CR RL AGNs observed with $H S T$ WFC3 as part of program GO13023. The RGB images are made using the WFC $3 F 140 W$ filter for the R channel, and the WFC3 UVIS F606W filter for both the G and B channels. the size of each image roughly corresponds to the field of view of WFC3 IR. The 10" reference corresponds to $84.6 \mathrm{Kpc}, 86.0 \mathrm{Kpc}, 83.8 \mathrm{Kpc}$, and $85.7 \mathrm{Kpc}$ for 3C 68.1, 3C 230, 3C 322, and 3C 297, respectively. In the figure we show one case from each outcome of the cluster classification scenarios. In the upper left panel the field of $3 \mathrm{C} 68.1$ is classified as a cluster on the basis of the presence of both an RS and an over density. The field of 3C 230 shows an RS but no over density. The field of 3C 322 shows an over density but no RS. Lastly, the field of 3C 297 shows neither an RS nor an over density. See Section 5 for details.

consequence of the fact that the models are reproducing the evolution of the stellar populations included in these galaxies since the first burst of star formation. At redshifts above $\sim 2$ a significant fraction of stars are still blue even in passive evolving ellipticals. Strikingly, a number of relatively bright ETGs in the field of 3C 257 are indeed blue and fall exactly in the region spanned by the models. In Figure 7 we show the WFC3 IR image of a region of $60^{\prime \prime} \times 40^{\prime \prime}$ in the vicinity of our target. 3C 257 is marked with a yellow circle and the three brightest ETGs are marked with green circles. These objects lie at a projected distance of $200-300 \mathrm{kpc}$ from the source. These galaxies may be similar to the star-forming blue ETGs associated with one proto-cluster and a group recently found by Mei et al. (2015) in the Hubble Ultra Deep Field.

Interestingly, a population of redder $(F 606 W-F 140 W \sim 3)$ objects is present in the field of this galaxy. However, these objects are not identified as ETGs. Visual inspection reveals that some of these galaxies, including the host galaxy of $3 \mathrm{C}$ 257, are close companions of the target (Figure 7). These objects display disturbed morphologies that are most likely indicating an active merger phase. We argue that these galaxies are reddened by the presence of a significant amount of dust. They are not recognized as ETGs because their morphology is very irregular. In the figure, these are the objects within about $10^{\prime \prime}$ of the target. Other red galaxies are located further away from the target and their association with the RG is less obvious. This hypothesis may be further tested using multi wavelength observations e.g., in the infrared or at radio wavelengths with ALMA. However, there is already strong evidence from Herschel observations that at least for the host galaxy of the radio source, extremely high star formation activity is present (Podigachoski et al. 2015).

Without spectroscopic information we cannot exclude that the blue ETGs in this field are a population of lower redshift galaxies. We are convinced that these objects are not foreground ETGs for two reasons: (i) a population of relatively bright blue ETGs is not commonly present in our sample; and (ii) their location is consistent with the prediction of the RS models. We speculate that this population of blue objects could be galaxies that will evolve into the RS galaxies observed in lower redshift clusters (Kodama et al. 1998, 2007).

Curiously, 3C 257 is one of the very few fields in our sample in which we do not detect a significant over density of sources. Since surface brightness depends on redshift as $(1+z)^{-4}$, this may result from incompleteness due to the detection threshold of our data. Another possible scenario that may explain the lack 


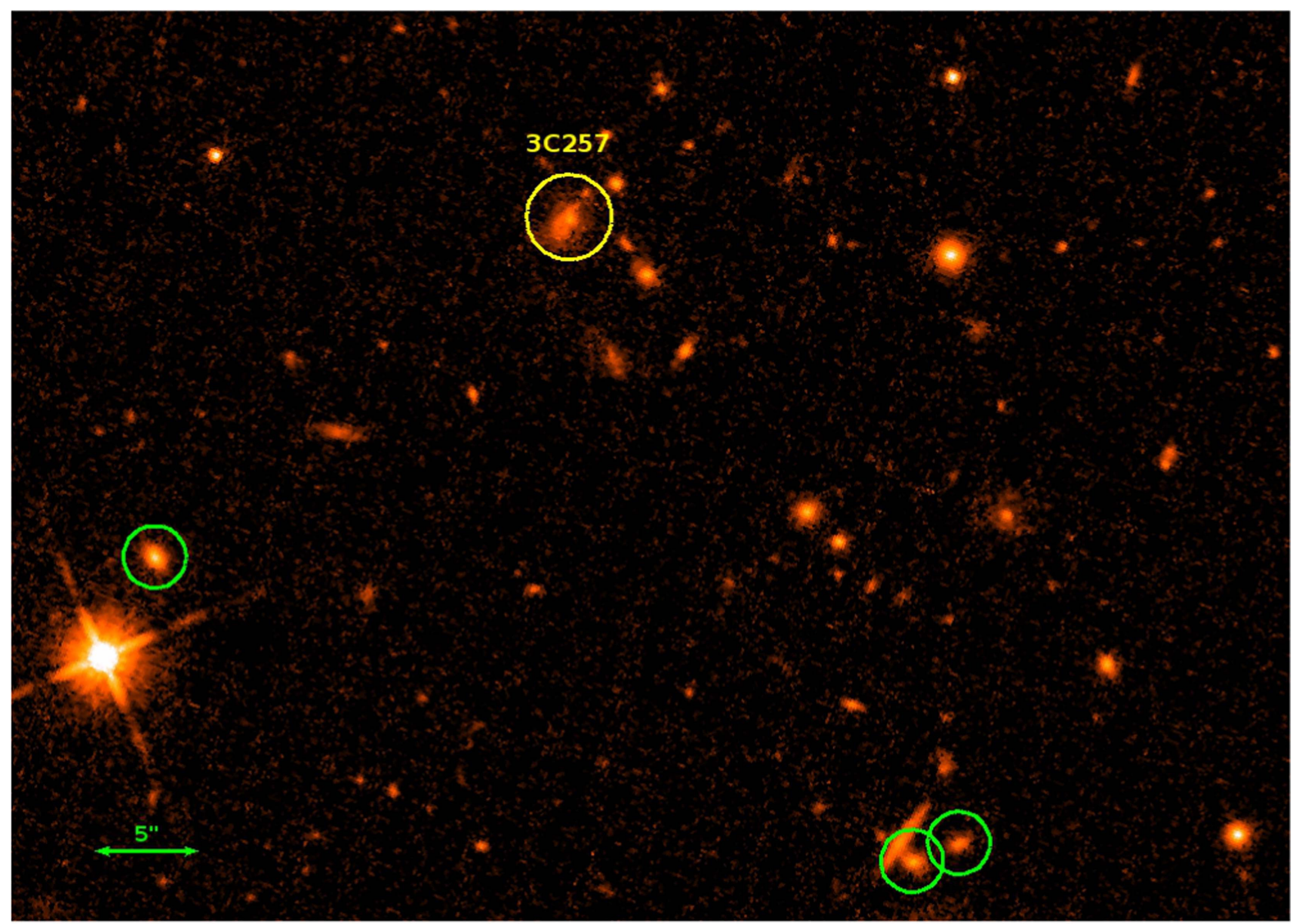

Figure 7. HST WFC3 IR image of a $60^{\prime \prime} \times 40^{\prime \prime}$ region surrounding $3 \mathrm{C} 257$. The $5^{\prime \prime}$ reference corresponds to $40.5 \mathrm{Kpc}$ at the redshift of the target. The yellow circle displays the target (3C 257) and the green circles show the three brightest ETGs in the field.
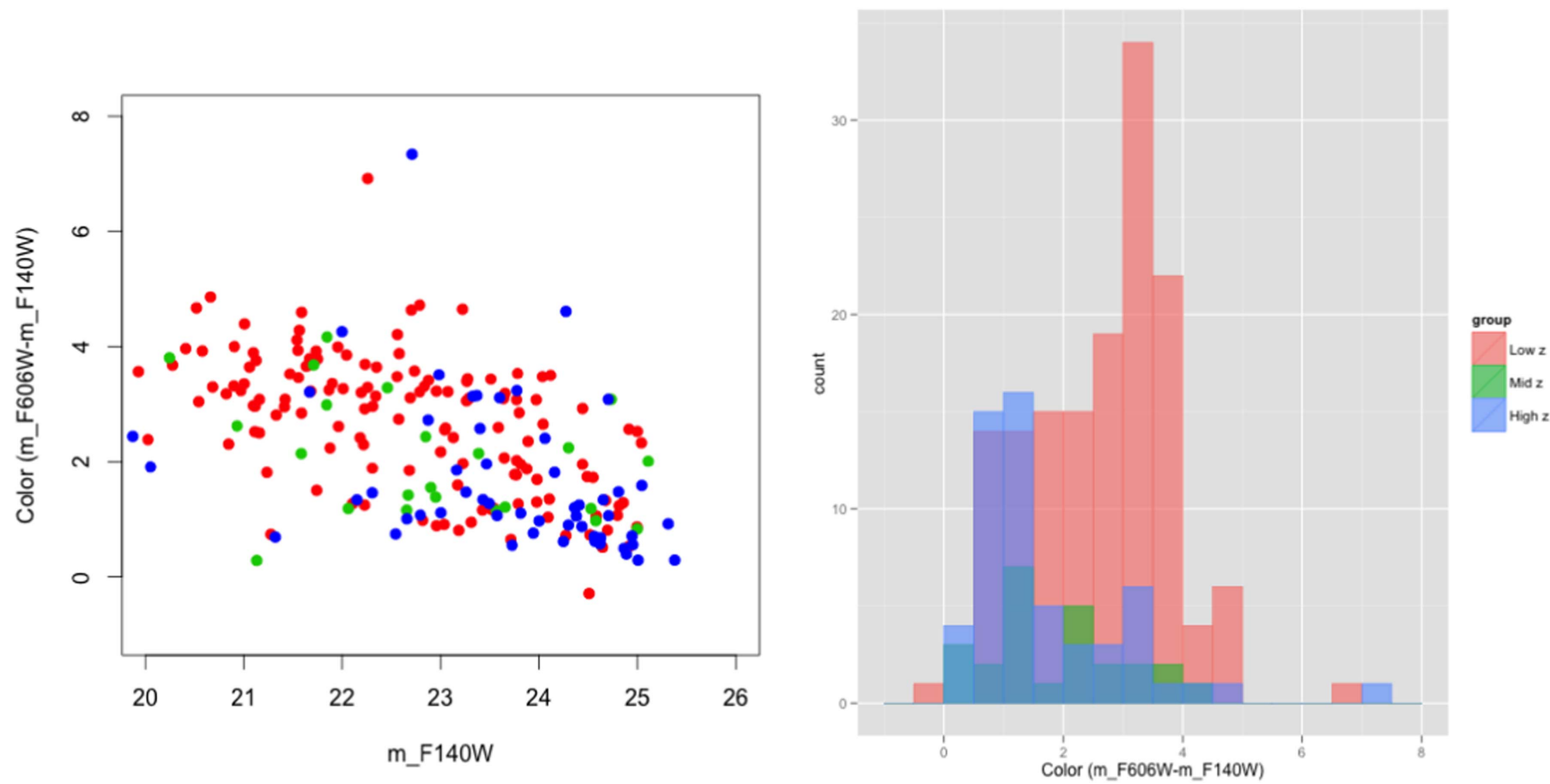

Figure 8. Left panel shows all of the ETGs identified in all of the 3CR fields. Different colors are assigned to different redshift bins. Red, green, and blue represent $z<1.3,1.3<z<1.75$ and $z>1.75$. Note that the redshifts correspond to the measured redshift of the RL AGNs at the center of each of the fields in which the ETG is located. The right panel shows the distribution of the color of the ETGs for each of the redshift bins previously specified. 
of a significant over density in this field is that at $z>2$ protoclusters are significantly more extended than the projected region of the sky covered by our observations, and less dense than similar structures at lower redshifts.

The presence of a trend between the color of the ETGs and redshift seems to be confirmed by the results shown in Figure 8. In the left panel we show the CMD for all of the ETGs identified in all of our 3C fields. Different colors identify the redshift range of the RG in which each ETG is found. Red is for $z<1.3$, green for $1.3<z<1.75$, and blue is for $z>1.75$. The lower redshift ETGs are clustered around a color $m_{F 606 W}-m_{F 140 W} \sim 3$. The higher redshift ETGs are instead around $\sim 1$. The intermediate objects are scattered of a large range of colors. This is even more clearly visible in the right panel of Figure 8, which shows histograms for the color distribution of the ETGs. This may be evidence that the ETGs in the fields of RGs between $z=1$ and 2.5 undergo dramatic evolution from star-forming to passive evolving RS galaxies.

\subsection{Location of $3 C R$ Targets in the CMDs}

The location of the hosts of the 3CR RGs in the CMDs with respect to the areas spanned by the RS models differs across the sample. We find that most of the RGs have a color ( F606W$F 140 W) \sim 2-3 \mathrm{mag}$. The evolutionary models for RS ETGs predict bluer colors as redshift increases. For the lowest redshift RGs $(z<1.3)$, the hosts are bluer than the RS models for the corresponding redshift. In the case of 3C 255 and 3C 297, with redshifts $z=1.35$ and $z=1.41$ respectively, we find that the host falls within the area spanned by the models. In the highest redshift RG in the sample, 3C 326.1, 3C 454.1, and 3C 257 $(z=1.83, z=1.84$, and $z=2.47$, respectively) the hosts' positions lie above the models in the CMD. The fact that the colors of the RG hosts do not follow the predictions of simple passive evolution directly implies that modeling the evolution of these objects requires additional parameters. For example, it is possible a major role in defining the stellar population is played by galaxy mergers, which appear to be closely connected to RL AGN activity (Chiaberge et al. 2015). Therefore the combined presence of dust and enhanced star formation associated with those events may be responsible for the observed color of each object at different redshifts. Detailed modeling of these effects is beyond the scope of this work, and will be performed in a forthcoming paper.

\section{CONCLUSIONS}

We studied the environments of 21 3CR RL AGNs in the redshift range $1.05<z<2.47$ using $H S T$ Snapshot observations taken with WFC3 UVIS and IR at rest-frame UV and optical wavelengths, respectively. We derived color versus magnitude diagrams for all of the fields in order to search for RSs defined by ETGs at the corresponding redshift of each radio source. We find that for seven targets the majority of the ETGs are located within the area spanned by RS passive evolution models. The second method classifies fields around the targets as being over dense if the object density is significantly higher than that of a control sample taken from 3D-HST GOODS-S. We determined that 3CR RL AGN at $z>1$ are associated with over-dense fields, on average. We also compare single targets with the average density of the control fields and we conclude that in 10 out of 21 cases the fields are over dense at more than $3 \sigma$. Our results are consistent with a fraction of $\sim 50 \%$ of objects being associated with clusters or groups, in agreement with previous results (e.g., Hill \& Lilly 1991; Best 2000).

For a number of objects the two methods do not agree on the cluster classification. In the cases where only the RS method classifies the field as a cluster, one possible explanation may be that the target resides in a lower density cluster or a group that includes a population of passively evolving ETGs. Alternatively, and particularly for the higher redshift objects, we may be missing a substantial number of faint cluster galaxies because of the inherent detection threshold of our data. Since most of our targets are clustered at $z<1.5$, it is difficult to statistically test if such an hypothesis is viable using our data. A larger number of high- $z$. $\mathrm{C}$ fields must be observed to allow for proper statistical analysis. This would allow us to both firmly establish whether the observed number density of galaxies around each radio source depends on redshift, and understand whether that is due to the detection limit of the survey. Alternatively, the lower number density of objects around more distant targets might be a result of the different dynamical status of those clusters.

For the opposite case in which an over density is present but there is no RS, the most likely explanation is that evolution is playing a role. A significant population of red objects are usually present but they are not classified as ETGs, likely because of their distorted morphologies, possibly as the result of mergers. The fact that our RS cluster candidates are all at $z<1.5$ seems to confirm that the redshift range of 3 to 1.5 is crucial for the formation of the RS and the evolution from proto-clusters to clusters of galaxies (Miley et al. 2006; Zirm et al. 2008; Galametz et al. 2010).

Perhaps the most important result of this work is the discovery of the presence of a population of blue ETGs in the field of the most distant objects in the 3CR catalog. This is apparent in particular for $3 \mathrm{C} 257$ at a redshift $z=2.47$. The location of these ETGs in the CMD is consistent with the predictions of our assumed simple passive evolution model for the redshift of the target. We also found evidence for a general trend with redshift for the color of the ETGs identified in all of our fields. The galaxies are typically bluer at higher redshifts, while they cluster around redder colors at $z<1.3$. This implies that these ETGs include a significant component produced by a younger and thus bluer stellar population that is rapidly evolving toward the redder part of the diagram. Clearly such a finding must be further investigated using narrow band imaging centered on Ly $\alpha$ (e.g., Venemans et al. 2004) and spectroscopy in order to both confirm these objects as cluster members and better study their stellar populations.

The authors thank Colin Norman for useful comments and insightful suggestions. J.P.K. and B.H. acknowledge support from HST-GO-13023.005-A. We thank the anonymous referee for constructive comments that improved the paper. This research has made use of the NASA/IPAC Extragalactic Database (NED) which is operated by the Jet Propulsion Laboratory, California Institute of Technology, under contract with the National Aeronautics and Space Administration. This work is based in part on observations taken by the 3D-HST Treasury Program (GO12177 and 12328). This work is based on observations made with the NASA/ESA HST, obtained from the Data Archive at the Space Telescope Science Institute, which is operated by the Association of Universities for 
Research in Astronomy, Inc., under NASA contract NAS 5-26555.

Facility: HST (WFC3).

\section{REFERENCES}

Anderson, J., \& Bedin, L. R. 2010, PASP, 122, 1035

Bertin, E., \& Arnouts, S. 1996, A\&AS, 117, 393

Best, P. N. 2000, MNRAS, 317, 720

Best, P. N., Kauffmann, G., Heckman, T. M., et al. 2005, MNRAS, 362, 25

Blakeslee, J. P., Franx, M., Postman, M., et al. 2003, ApJL, 596, L143

Blandford, R. D., \& Znajek, R. L. 1977, MNRAS, 179, 433

Brammer, G. B., van Dokkum, P. G., Franx, M., et al. 2012, ApJS, 200, 13

Calderone, G., Ghisellini, G., Colpi, M., \& Dotti, M. 2013, MNRAS, 431, 210

Castignani, G., Chiaberge, M., Celotti, A., \& Norman, C. 2014, ApJ, 792, 113

Castignani, G., Haardt, F., Lapi, A., et al. 2013, A\&A, 560, A28

Chiaberge, M., Gilli, R., Lotz, J. M., \& Norman, C. 2015, ApJ, 806, 147

Chiaberge, M., \& Marconi, A. 2011, MNRAS, 416, 917

Donzelli, C. J., Chiaberge, M., Macchetto, F. D., et al. 2007, ApJ, 667, 780

Dunlop, J. S., McLure, R. J., Kukula, M. J., et al. 2003, MNRAS, 340, 1095

Galametz, A., Stern, D., De Breuck, C., et al. 2012, ApJ, 749, 169

Galametz, A., Vernet, J., De Breuck, C., et al. 2010, A\&A, 522, A58

Ghisellini, G., Tavecchio, F., Maraschi, L., Celotti, A., \& Sbarrato, T. 2014, Natur, 515, 376

Gladders, M. D., \& Yee, H. K. C. 2000, AJ, 120, 2148

Hilbert, B., Chiaberge, M., Kotyla, J. P., et al. 2016, ApJS, in press arXiv: (1605.03196)

Hill, G. J., \& Lilly, S. J. 1991, ApJ, 367, 1

Kodama, T., Arimoto, N., Barger, A. J., \& Arag'on-Salamanca, A. 1998, A\&A, 334, 99

Kodama, T., Tanaka, I., Kajisawa, M., et al. 2007, MNRAS, 377, 1717
Kotulla, R., Fritze, U., Weilbacher, P., \& Anders, P. 2009, MNRAS, 396, 462 Krist, J. E., Hook, R. N., \& Stoehr, F. 2011, Proc. SPIE, 8127, 81270J

Kron, R. G. 1980, ApJS, 43, 305

Laor, A. 2000, ApJL, 543, L111

Lee, H., Bell, E. F., \& Somerville, R. S. 2008, in IAU Symp. 255, LowMetallicity Star Formation: From the First Stars to Dwarf Galaxies (Cambridge: Cambridge Univ. Press), 100

Long, K., Wheeler, T., \& Bushouse, H. 2011, WFC3 Instrument Science Rep. 2011-09 (Baltimore: STScI)

Mao, M. Y., Owen, F., Duffin, R., et al. 2015, MNRAS, 446, 4176

Mei, S., Scarlata, C., Pentericci, L., et al. 2015, ApJ, 804, 117

Miley, G., \& De Breuck, C. 2008, A\&ARv, 15, 67

Miley, G. K., Overzier, R. A., Zirm, A. W., et al. 2006, ApJL, 650, L29

Peng, C. Y., Ho, L. C., Impey, C. D., \& Rix, H.-W. 2010, AJ, 139, 2097

Podigachoski, P., Barthel, P. D., Haas, M., et al. 2015, A\&A, 575, A80

Prestage, R. M., \& Peacock, J. A. 1988, MNRAS, 230, 131

R Core Team 2014, R: A Language and Environment for Statistical Computing (Vienna: R Foundation for Statistical Computing)

Rosati, P., Borgani, S., \& Norman, C. 2002, ARA\&A, 40, 539

Siemiginowska, A., Burke, D. J., Aldcroft, T. L., et al. 2010, ApJ, 722, 102

Spinrad, H., Marr, J., Aguilar, L., \& Djorgovski, S. 1985, PASP, 97, 932

Stanford, S. A., Eisenhardt, P. R., Dickinson, M., Holden, B. P., \& De Propris, R. 2002, ApJS, 142, 153

Sunyaev, R. A., \& Zeldovich, Y. B. 1972, CoASP, 4, 173

van Dokkum, P. G. 2001, PASP, 113, 1420

Venemans, B. P., Röttgering, H. J. A., Overzier, R. A., et al. 2004, A\&A, 424, L17

Wilson, A. S., \& Colbert, E. J. M. 1995, ApJ, 438, 62

Wylezalek, D., Galametz, A., Stern, D., et al. 2013, ApJ, 769, 79

Zirbel, E. L. 1996, ApJ, 473, 713

Zirbel, E. L. 1997, ApJ, 476, 489

Zirm, A. W., Stanford, S. A., Postman, M., et al. 2008, ApJ, 680, 224 